\title{
Breast cancer surgical treatment choices in Newfoundland and Labrador, Canada: Patient and surgeon perspectives
}

\author{
Holly Etchegary, ${ }^{1}$ Elizabeth Dicks, ${ }^{1}$ Farah McCrate, ${ }^{2}$ Erin Powell, ${ }^{2}$ Joanne Chafe, ${ }^{3}$ \\ Rebecca Roome, ${ }^{4}$ Charlene Simmonds ${ }^{5}$ \\ ${ }^{1}$ Faculty of Medicine, Memorial University, ${ }^{2}$ Cancer Care Program, Eastern Regional Health \\ Authority, ${ }^{3}$ Centre for Nursing Studies, Eastern Regional Health Authority, ${ }^{4}$ Patient/community \\ representative, ${ }^{5}$ Health Research Unit, Faculty of Medicine, St. John's, Newfoundland, Canada
}

\begin{abstract}
Significance for public health
Cancer treatment choices are complex decisions, affected by numerous clinical, demographic and social variables. Research is needed to understand why women from Newfoundland and Labrador have the highest rate of mastectomy in Canada. Greater understanding of the influences on women's surgical choices may help encourage informed decisions amongst women and physicians and promote active communication about treatment, benefits relevant to all jurisdictions and health authorities. Further, if factors such as geographic proximity to treatment facilities are associated with treatment decisions, this information is important for public healthcare spending and service planners. Finally, it is our hope the findings from this project could help improve the screening process and surgical care of breast cancer patients in Newfoundland and Labrador.
\end{abstract}

\section{Abstract}

Background. Breast cancer remains the second-leading cause of cancer death among Canadian women. Treatment for breast cancer often includes surgery. Many women have a choice between mastectomy (MT; removal of the entire breast) or breast conserving surgery (BCS; removal of the tumour and some noncancerous breast tissue) followed by radiation. However, Newfoundland and Labrador consistently has a higher rate of mastectomies than the rest of Canada. In this project, we aim to better understand that trend.

Design and methods. A multi-method design was chosen. Surgical treatment data kept by the province will be examined to describe the number and types of breast cancer surgeries over time. Second, we will hold focus groups with women around the province who have made surgical treatment choices to explore influences on their decisions. Finally, semi-structured interviews with breast cancer surgeons and surgical residents will explore their opinions on surgical treatment choices.

Expected impact for public health. Cancer treatment choices are complex decisions, affected by clinical, demographic and social variables. Understanding why women from Newfoundland and Labrador have the highest rate of mastectomy in Canada is critical to ensure they are receiving appropriate screening and care. Greater understanding of the influences on women's surgical choices may encourage informed decisions amongst women and physicians and promote active communication about treatment, benefits relevant to all jurisdictions and health authorities. Further, if factors such as geographic proximity to treatment facilities are associated with treatment decisions, this information is important for public health screening and service planners.

\section{Introduction}

Breast cancer remains the most common cancer diagnosed among Canadian women and the second-leading cause of cancer death. ${ }^{1}$ The Canadian Breast Cancer Foundation reports one quarter of new female cancer cases in 2016 was due to breast cancer (www.cbcf.org). Improvements in breast cancer treatment, however, suggest that early stage breast cancer has a favourable prognosis. ${ }^{1,2}$ Most women diagnosed with non-metastatic breast cancer are candidates for surgery, whether breast conserving surgery (BCS, also known as lumpectomy) followed by radiation therapy, or mastectomy (MT). ${ }^{1}$ In BCS, the tumour is removed along with a margin of non-cancerous breast tissue, while MT involves removal of the entire breast. BCS is less invasive than MT, associated with better psychological outcomes, and if followed by radiation, has comparable survival. ${ }^{3}$ Thus, guidelines suggest that if $\mathrm{BCS}$ is not contraindicated on clinical grounds, women with breast cancer should be offered a choice between BCS and MT., ${ }^{2,4}$ It might be assumed that eligible women would choose the less radical $\mathrm{BCS}$. However, breast units worldwide display varying surgical practice, ${ }^{1}$ that is not explained by case mix. ${ }^{5} \mathrm{~A}$ panCanadian study ${ }^{2}$ revealed wide inter-provincial variation in surgical treatment choices. The crude MT rate was 39\%, but this varied from $26 \%$ in Quebec to $69 \%$ in Newfoundland and Labrador. A special focus report on breast cancer in $\mathrm{Canada}^{1}$ revealed that while $80 \%$ of breast cancer cases in Canada in 2010 were diagnosed with early disease, NL had a higher percentage (27\%) diagnosed with advanced disease (Stage 3 or 4). It is unlikely, however, that stage distribution alone accounts for the variation in surgical management of breast cancer in Canada as there is a fairly comparable stage distribution across provinces. ${ }^{1,2}$ A number of socio-demographic variables influence women's surgical choices. For example, age, income and education have all been associated with surgical choice, with women of higher income and education levels more likely to choose BCS. ${ }^{6,7}$ The literature regarding the effect of age is mixed, due in part to heterogeneity among studies in age cutoffs and the year in which studies are conducted. ${ }^{6}$ It is suggested that age alone is not a significant predictor of surgical choice. ${ }^{6}$ Women's attitudes and beliefs about cancer recurrence, chance of survival, body image and sexuality, as well as radiation therapy have also been associated with cancer surgical decisions. ${ }^{8-}$ ${ }^{11}$ Clinical factors such as tumour size and stage, ${ }^{7}$ geographic area (rural vs. urban), ${ }^{1}$ the availability of radiation facilities, and clinician advice also affect women's surgical decisions. ${ }^{11,12}$ Canadian women with longer travel times to a radiation facility were more likely to undergo MT. ${ }^{2}$ The recent special focus report on breast cancer in $\mathrm{Canada}^{1}$ revealed that until travel time exceeds 40 minutes, the MT rate is constant, after which it increases by $7 \%$. The 
MT rate increases further with additional minutes of travel time. It is noted that whether these patterns are due to travel-related barriers or other factors associated with rural living is currently unknown. Finally, an important factor with a strong influence on women's treatment choices is the preference of their surgeon or oncologist. ${ }^{11}$ This is important in light of recent support for shared decision-making in healthcare contexts and evidence-informed patient choice. Specifically, evidence suggests that women whose preferred level of involvement in treatment decisions is met have better quality of life and satisfaction with their treatment decisions. ${ }^{12,13}$

\section{Significance}

Greater understanding of the influences on women's surgical choices may help encourage informed decisions amongst women and physicians and promote active communication about treatment. Further, if factors such as geographic proximity to treatment facilities are associated with treatment decisions, this information is critical for healthcare spending and service planning. Finally, given the higher rate of mastectomies in NL breast cancer patients, ${ }^{2}$ research is needed to understand this pattern and ensure care is offered appropriately.

\section{Overall study design}

We have assembled a research team composed of academic researchers, epidemiologists, healthcare professionals and (former) breast cancer patients. In this three-year study, we will use quantitative and qualitative methods to address study objectives and questions. The study protocol has been approved by the provincial Health Research Ethics Authority (Reference \#2016.023).

\section{Key study objectives}

To document the number of breast cancer surgeries in NL, including information on tumor stage (the latter not available in the recent Canadian report, but known to affect whether BCS or MT is offered to patients). ${ }^{2}$

To explore patients' and surgeons' perceptions of the factors influencing the choice of BCS with radiation therapy or MT.

\section{Research questions and hypotheses}

What is the rate of BCS and MT in NL over a given time period (the highest quality data is expected to be for the last 5 years)? Based on the recent Canadian report ${ }^{2}$, we expect a higher MT rate compared to BCS.

What clinical and non-clinical factors are associated with these rates? (e.g., tumour stage and size, age, rural vs. urban). We hypothesize that tumour stage and living area will be associated with surgical treatment, such that a later stage tumour is associated with MT, as is living a further distance from a radiation facility.

What factors do patients and surgeons perceive affect surgical treatment choice in breast cancer? We have no specific hypotheses for this qualitative item, but suspect clinicians will focus on clinical characteristics of the tumour and chance of survival, while women will invoke a wider range of influences (e.g., family concerns, cancer fear, body image concerns, etc.).

\section{Materials and Methods}

\section{Quantitative}

First, we propose a quantitative, retrospective cohort study using surgery data maintained in the Discharge Abstract Database at the Newfoundland and Labrador Centre for Health Information to describe the rates of BCS and MT in NL. We will extract other clinical and non-clinical information from the provincial Tumour Registry (e.g., tumour stage and size, age, living area). Regression analyses will be used to predict treatment decision (BCS or MT). Following the recent pan-Canadian study, ${ }^{2}$ a logistic regression model can be fit with independent variables such as tumour stage, age, travel time to nearest radiation facility and other available tumour and demographic variables. Coefficients derived from the model will be used to calculate the probability of MT or BCS for each woman. The expected provincial rate of MT or BCS is based on the sum of the probabilities of MT or BCS for all women living in NL. Adjusted rates of MT or BCS can then be calculated as follows: (crude MT or BCS rate/predicted MT or BCS rate) $\times$ the crude rate for Canada. ${ }^{1,2}$

\section{Qualitative}

\section{Semi-structured interviews with surgeons and surgical residents}

In parallel to the review of administrative data, we will hold focus groups with former and current breast cancer patients and conduct semi-structured interviews with surgeons and residents. Interviews were chosen with surgeons and residents as it was thought arranging group discussions would be difficult in light of varying and busy surgical schedules. Breast surgeons around the province will be identified through the team's healthcare contacts, as well as with the assistance of the Newfoundland and Labrador Medical Association and/or the College of Physicians and Surgeons of Newfoundland and Labrador. Eligible surgeons and residents will be sent a study information invitation letter through the email/mailing lists of relevant provincial organizations and/or the Faculty of Medicine at Memorial University. Eligibility criteria include: 1 . Be a practicing general surgeon who performs breast surgeries in any of the four health regions of the province; 2.Or, be a general surgical resident at the time of the study recruitment; and 3. Speak English. Through the team's contacts, we will use snowballing recruitment to generate additional names (e.g., we will ask each surgeon to recommend another to whom a study invite can be sent). Flexibility in interview scheduling and modality (e.g., telephone, face-to-face, online) will be employed in order to accommodate as many surgeons/residents as possible. Our goal is to collect data in each of the province's four health authorities in order to include views from all areas of our province. Prior qualitative research of team members suggests that 15-20 interviews tend to achieve data saturation (i.e., the point at which no new additional information arises in interviews). We aim for 15-20 interviews in total, spread across the health authorities (Table 1).

\section{Focus groups with women who have made breast cancer surgical decisions}

To recruit women who have made surgical treatment decisions for breast cancer, we will ask participating surgeons to identify eligible women. Women will be sent a study invite letter and invited to call the research team if they are interested. We will advertise the study in newspapers, community cancer organizations (e.g., cancer fundraisers such as the provincial Relay for Life), and in conjunction with the Dr. Bliss Murphy Cancer Centre (e.g., study ads can be included in mailouts, newsletters, posted at Daffodil Place and support groups). We aim to conduct focus groups $(n=12)$ with women from each of the four health authorities. Prior work of the team (both in breast cancer and other health research) suggests two focus groups per region (with 5-8 participants) should ensure data saturation. We plan to hold group sessions in cities and towns such as the capital city of St. John's, as well as the towns of Grand 
Falls-Windsor, Gander, Corner Brook and two sites in Labrador. Team members (in particular Dicks) have previously held focus groups with breast cancer patients in St. John's and found them to be an acceptable way to discuss treatment options and choices, with six women per group being optimal. Focus groups are also appropriate when participants share similar experiences and a goal is to generate understanding of a complex phenomenon at an affordable cost (Table 2).

\section{Qualitative data analysis}

In the main, qualitative description ${ }^{14}$ will be employed to summarize the data pertaining to breast cancer surgical treatment choices. This form of naturalistic inquiry makes no theoretical assumptions about the data. Its goal is to present the data in the language of participants, without aiming to interpret the data in more theoretical ways. The end result is a comprehensive summary of the event in question. ${ }^{15}$ Qualitative description was a logical choice for analysing study data as a key goal is to provide a comprehensive account of the factors influencing breast cancer surgical treatment choices from the perspectives of both women and healthcare professionals. Transcripts will be read and re-read several times by three team members (HE, ED, and RR; the latter two members have made surgical breast cancer treatment decisions and will bring that lens to analysis). Interview data will be isolated and organized around interview topics and utilized to identify and index emerging categories and themes for particular questions. These investigators will separately read and re-read the isolated data, and use the method of constant comparison to inductively sub-code the data relevant to surgical treatment decisions. ${ }^{14-15}$ Essentially, data will be compared between and within transcripts to establish analytical categories and themes, ${ }^{14-15}$ with a constant shifting back and forth between and within transcripts to compare

Table 1. Semi-structured interview guide for use with breast cancer surgeons and residents. A recent pan-Canadian study reported that Newfoundland and Labrador had the highest rates of mastectomies in Canada.

\begin{tabular}{|c|c|}
\hline Themes & Questions and probes for discussion \\
\hline Comment on statement above & $\begin{array}{l}\text { Does this finding surprise you? (probes - does that correspond with your clinical experience? } \\
\text { Would you agree with the finding? Why do you think that might be?) }\end{array}$ \\
\hline Mastectomy treatment & What factors would lead you to recommend mastectomy to a breast cancer patient? \\
\hline Breast conserving surgical treatment & $\begin{array}{l}\text { What factors would lead you to recommend breast conserving surgery instead? } \\
\text { (Probes - would this always involve radiation therapy as well? Are there non-clinical factors that might } \\
\text { influence your surgical recommendation?) }\end{array}$ \\
\hline Perceptions of women's surgical choices & $\begin{array}{l}\text { Do you think women should be involved in decision making regarding their surgical treatments? If so how? } \\
\text { (probes - Do you think women want to be involved in decision making regarding their surgical treatments? } \\
\text { If so, to what extent? What do you think of the current recommendation for 'shared decision-making' } \\
\text { among patients and clinicians?) }\end{array}$ \\
\hline $\begin{array}{l}\text { Reflections on the factors influencing } \\
\text { women's surgical choices }\end{array}$ & $\begin{array}{l}\text { Which factors do you feel most influence women's choices about MT or BCS? } \\
\text { (probe - do you think there is a most important factor for women in making the decision to undergo MT } \\
\text { or BCS?) }\end{array}$ \\
\hline Need for education and tools & $\begin{array}{l}\text { Do you think there is any benefit to developing a decision aid or some kind of tool for women to help them make } \\
\text { choices about surgery related to breast cancer? (probe - what might this tool look like? } \\
\text { What exactly would be helpful?) }\end{array}$ \\
\hline
\end{tabular}

Table 2. Focus group guide with women who have made surgical treatment choices.

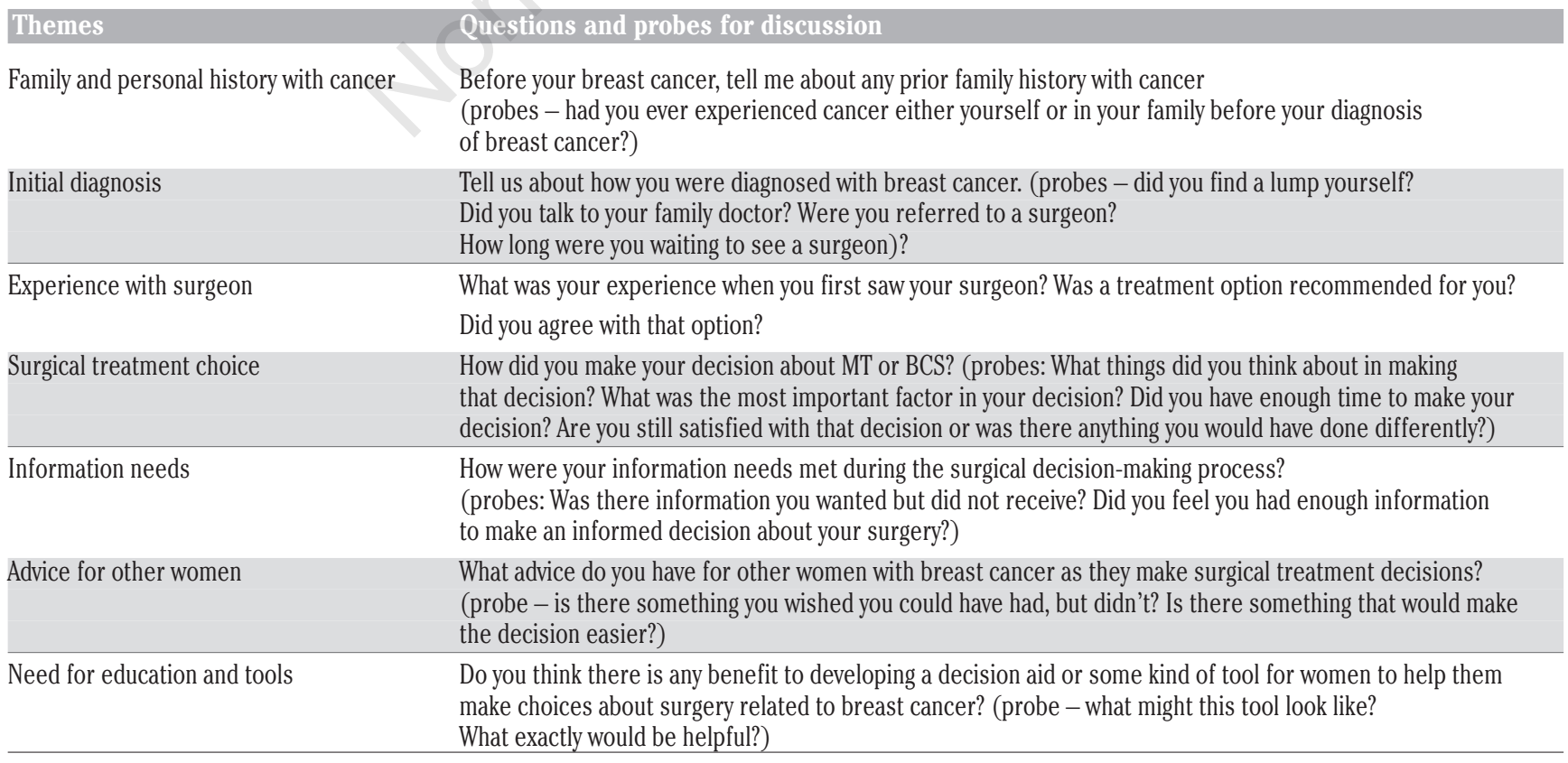


the perceptions and experiences of participants. When investigators complete their separate analyses, they will meet to review and reach consensus on categories and themes before sharing results with other members of the team for validation.

\section{Ethical issues}

Usage of all data held by NLCHI and the NL Tumour Registry follows strict ethical guidelines regarding linkage, de-identification and access. Data will be de-identified, so only provincial-level data will be reported, with no personal identifiers. The collection and storage of qualitative data will follow best ethical practices (e.g., participants will be advised that participation is voluntary and given a pseudonym in any reports produced from the data). Breast cancer participants will also be advised that taking part in the study will in no way affect their healthcare. Further, one of team members (JC) is an experienced counsellor and will be available for further discussion with women should distress occur.

\section{Engagement of stakeholders/knowledge translation.}

To further engage with stakeholders, we will use town hall meetings and academic speaking opportunities to present findings. We will aim to do this before finalizing the summary report of the study, so as to include any additional comments/discussions from town halls. Following the town halls, we will finalize the summary report of the study and provide participants with a copy, and also make copies of the summary available to the provincial cancer organizations and treatment centres. We will post a link to the study summary on both Memorial University's and provincial health authorities' websites. We will contact community organizations who helped with recruitment and offer to send a copy to their members. Finally, study findings will be published in academic journals and presented at relevant conferences.

Correspondence: Holly Etchegary, Faculty of Medicine, Memorial University, Craig L. Dobbin Centre for Genetics, Rm 4M210, St. John's, NL, A1B 3V6, USA.

Tel.: + 1.709.864.6605

E-mail: holly.etchegary@med.mun.ca

Key words: breast cancer, mastectomy, lumpectomy, surgery, qualitative. Contributions: HE and ED conceived of the study, are co-PIs for the project and have overall responsibility for the entire study. All authors discussed and finalized the study protocol. All authors have been involved in study recruitment and data collection across the different phases of the project. HE, ED, and FM are primarily responsible for data analysis, with review and input from all team members where appropriate. HE drafted the study protocol and all authors read and revised it for important intellectual content; all authors read and approved the final version.

Conflict of interest: the authors declare no potential conflict of interest. Funding: provided by the Enhancing Healthcare in Newfoundland and Labrador competition, administered through the Newfoundland and Labrador Centre for Health Information, St. John's, Newfoundland, Canada. We are grateful to the surgeons and women who have taken part in the study thus far.

Received for publication: 9 March 2017.

Accepted for publication: 19 July 2017.

(C) Copyright H. Etchegary et al., 2017

Licensee PAGEPress, Italy

Journal of Public Health Research 2017;6:867

doi:10.4081/jphr.2017.867

This work is licensed under a Creative Commons Attribution NonCommercial 4.0 License (CC BY-NC 4.0).

\section{References}

1. Canadian Partnership Against Cancer. Breast cancer control in Canada: a system performance special focus report. Toronto, Canada. 2012. Available from: https://content.cancerview.ca/ download/cv/quality_and_planning/system_performance/documents/breastcancercontrolreppdf?attachment $=0$

2. Porter G, Wagar B, Bryant H, et al. Rates of breast cancer surgery in Canada from 2007/08 to 2009/10: retrospective cohort study. CMAJ Open 2014;2.

3. Veronesi U, Cascinelli N, Mariani L, et al. Twenty-year follow up of a randomized study comparing breast conserving surgery with radical mastectomy for early breast cancer. N Engl J Med 2002; 16:1227-32.

4. Whelan T, Olivotta I, Levine M, Canadian Breast Screening Initiative, Steering Committee on Clinical Practice Guidelines for the Care and Treatment of Breast Cancer. Clinical Practice guidelines for the care and treatment of breast cancer: breast radiotherapy after breast conserving surgery (summary of the 2003 update). Hamilton, ON. 2003. Available from: http:/www.cmaj.ca/content/172/10/1319.full.pdf

5. Caldon L, Walters S, Reed J, et al. Case-mix fails to explain variation in mastectomy rates: management of screen-detected breast cancer in a UK region 1997-2003. Br J Cancer 2005;92:55-9.

6. MacBride M, Neal L, Dilaveri C, et al. Factors associated with surgical decisions making in women with early-stage breast cancer: a literature review. J Women's Health 2013;22:236-42.

7. Bellavance E, Kesmodel S. Decision-making in the surgical treatment of breast cancer: factors influencing women's choices for mastectomy and breast conserving surgery. Front Oncol 2016;6:74.

8. Swainston K, Campbell C, van Wersch A, Durning P. Treatment decision making in breast cancer: a longitudinal exploration of women's experience. Br J Health Psych 2011;17:155-70.

9. Osborn G, Hodin M, Drew P, et al. Patient demographics and treatment for early breast cancer: an observational study. Breast 2006;15:377-81.

10. Gilligan M, Kneusel R, Hoffmann R, et al. Persistent differences in sociodemographic determinants of breast conserving treatment despite overall increased adoption. Med Care 2002;40:181-9.

11. Aziato L, Clegg-Lamptey J. Breast cancer diagnosis and factors influencing treatment in Ghana. Health Care Women Inter 2015;36:543-57.

12. Schonberg M, Birdwell R, Bychkovsky B, et al. Older women's experience with breast cancer treatment decisions. Breast Cancer Res Treat 2014;145:211-23.

13. O'Leary K, Estabrooks C, Olson K, Cumming C. Information acquisition for women facing surgical treatment for breast cancer: influencing factors and selected outcomes. Pat Educ Couns 2007;69:5-19.

14. Sandelowski M. Whatever happened to qualitative description? Res Nurs Health 2000;23:334-30.

15. Pope C, Ziebland S, Mays N. Analysing qualitative data. BMJ 2000;320:114-6. 